\title{
TRATAMENTO CIRÚRGICO DAS FRATURAS SUBCONDILARES: AVALIAÇÃO DA ABORDAGEM RETROMANDIBULAR DE HINDS
}

\author{
SURGICAL TREATMENT FOR SUB-CONDYLAR FRACTURES: ANALYSIS OF HINDS \\ RETROMANDIBULAR APPROACH
}

\author{
Ângela Alves de Aguiar Goto ${ }^{1}$; Sylvio Alves de Aguiar²; \\ Odilon Victor Porto Denardin ${ }^{3}$; Abrão Rapoport, ECBC- SP
}

\begin{abstract}
RESUMO: Objetivo: Avaliar retrospectivamente os resultados dos procedimentos terapêuticos cirúrgicos, através da abordagem retromandibular de Hinds, no tratamento das fraturas subcondilares. Método: o protocolo foi observacional, transversal, retrospectivo com inclusão de 31 casos divididos em quatro grupos, de acordo com a lateralidade, desvio da fratura e associação de fraturas em outros sítios. O sucesso terapêutico foi classificado de acordo com a necessidade de tratamento fisioterápico e bloqueio, permanência de desvios ou lateralidade de abertura bucal, amplitude reduzida de abertura bucal (menos de 40 mm), presença de dor moderada à intensa ou ausência de redução da fratura e instabilidade da osteossíntese. Para avaliação dos fatores determinantes de sucesso foram incluídas as variáveis: gênero, idade, causa da fratura, lateralidade, desvio, fraturas associadas e localização das fraturas. O método estatístico utilizou tabelas de associação cruzada com análise pelo teste não paramétrico do qui-quadrado. Em todas as situações foi arbitrado um valor de significância de 5\% ( $\mathrm{p}<0,05)$. Resultados: Encontrou-se uma resposta de sucesso total em 19 casos $(61 \%)$ e sucesso parcial em 12 casos (39\%). Não foram encontradas associações entre a resposta cirúrgica e as variáveis gênero, idade, causa da lesão e presença de fratura associada. Os casos com desvios laterais e bilateralidade da lesão obtiveram maior frequiência de sucessos parciais. Conclusão: Na amostra estudada, a abordagem retromandibular de Hinds para o tratamento das fraturas subcondilares, na maioria dos casos, teve sucesso total após o procedimento (Rev. Col. Bras. Cir. 2007; 34(5): 303-309).
\end{abstract}

Descritores: Fraturas do côndilo mandíbular; Cirurgia.

\section{INTRODUÇÃO}

A região condilar é acometida em $35 \%$ de traumatismos mandibulares, sendo na maioria das vezes resultantes de impactos (acidentes automobilísticos, de bicicleta e atropelamentos) sobre a região de sínfise e parassínfise mandibular ${ }^{1}$. O diagnóstico muitas vezes passa despercebido principalmente quando o paciente é atendido por profissionais não especializados.

O côndilo mandibular é protegido pelo processo zigomático do osso temporal, pelos músculos e ligamentos sobre a articulação. Por esta razão, o colo do côndilo é a parte mais delgada da mandíbula e está sujeito a fraturas causadas por ação de força indireta, tanto por impactos anteriores quanto laterais. Um trauma violento sobre a região sinfisária pode resultar em fratura da sínfise por força direta e fratura bilateral subcondiliana por força indireta. Se o trauma for dirigido a um lado mandibular, poderá causar fratura no local do impacto e fratura de colo de côndilo no lado oposto.

Os traumatismos condilianos podem ser classificados baseando-se no traço de fratura, localizado na região condilar alta, média ou baixa, relacionados respectivamente, com o nível de inserção do músculo pterigóideo lateral, seja acima ou abaixo do mesmo e com a base do crânio, podendo ser unilaterais ou bilaterais ${ }^{2}$.
Clinicamente, um paciente portador de fratura condilar, pode apresentar ${ }^{3-5}$ (1) evidência de trauma facial, principalmente na área de mandíbula e especialmente na sínfise, (2) edema e dor localizada na região de articulação temporomandibular (ATM), (3) limitação da abertura bucal, (4) desvio, ao abrir a boca em direção ao lado fraturado, (5) mordida aberta posterior, no lado contralateral, (6) mudança da oclusão no lado fraturado, com possível mordida cruzada, (7) presença de sangue no canal auditivo, (8) dor à palpação no lado fraturado, (9) ausência de movimento condilar à palpação, tanto transmeato quanto lateralmente sobre o côndilo, (10) em protrusão, desvio mandibular em direção ao lado fraturado, (11) inabilidade do paciente de excursionar a mandíbula no lado fraturado, (12) dificuldade de excursões e de protrusão da mandíbula e (13) ocorrência de mordida aberta, geralmente, em fraturas subcondilares bilaterais.

Fraturas condilares altas, apresentando grandes deslocamentos, são reduzidas cirurgicamente através de abordagens pré-auriculares ${ }^{6}$. Esta é indicada por apresentar proximidade ao fragmento fraturado e muito questionada quanto à possibilidade de seccionar ou traumatizar mecanicamente o nervo facial, podendo ocasionar inúmeras seqüelas ${ }^{7,8}$.

Fraturas medianas e baixas na região condilar podem ser reduzidas através de abordagem submandibular e retromandibular de Hinds ${ }^{6}$. Esta última por ser paralela ao ramo

\footnotetext{
1. Mestranda do Curso de Pós-Graduação em Ciências da Saúde do Hospital Heliópolis, Hosphel, São Paulo.

2. Chefe do Serviço de Cirurgia e Traumatologia do Hospital Samaritano, São Paulo.

3. Docente Permanente do Curso de Pós-Graduação em Ciências da Saúde do Hospital Heliópolis, Hosphel, São Paulo.

Recebido em 15/02/2007

Aceito para publicação em 17/04/2007
}

Conflito de interesses: nenhum

Fonte de financiamento: nenhuma

Trabalho realizado no Hospital Samaritano, São Paulo - SP, Brasil. 
mandibular, proporciona boa visão da fratura e pouca morbidade em relação ao nervo facial e aos vasos sanguíneos. Várias maneiras de fixação e redução são descritas pelos autores $^{4,6,9-12}$, como aplicação de uma ou duas miniplacas de titânio, parafusos tipo Lag Screw, fios de Kischner e placas compressivas.

A ausência de estudos sobre a melhor abordagem para o tratamento das fraturas subcondilares, justificou o emprego da abordagem retromandibular de Hinds, seguido de acompanhamento para avaliação dos resultados.

\section{MÉTODO}

Foram avaliadas as abordagens retromandibulares de Hinds, realizados em 31 indivíduos, no período de janeiro de 2002 a setembro de 2004. O estudo foi aprovado no Comitê de Ética na Pesquisa do Hospital Heliópolis, São Paulo, sob o número 434 em 13.12.2005.

Desses 31 pacientes atendidos, 16 eram do gênero feminino, e 15 do gênero masculino, com idades variando entre 12 e 63 anos. Etiologicamente, tais fraturas originaram-se de quedas, agressões físicas e acidentes automobilísticos.

Foram solicitadas tomografias computadorizadas (TC) com cortes coronais e em três dimensões para comprovação do diagnóstico clínico de fraturas subcondilares e avaliação dos desvios e fraturas associadas. Para este estudo, foram classificadas como subcondilares, fraturas localizadas próximo à incisura mandibular ou logo abaixo da mesma. Os desvios diagnosticados pelas tomografias, dividiram-se em mediais (Figura 1) e laterais.

Como todos os pacientes apresentaram desvios a partir de $30^{\circ}$ em suas fraturas, confirmados por tomografia computadorizada, associados a desordens oclusais, foi indicado o tratamento cirúrgico.

A abordagem retromolar de Hinds para fraturas subcondilares consiste em incisão de 2,5 a 3,0 cm em região de pele atrás e abaixo do ramo mandibular ${ }^{13}$, divulsionando tecidos musculares, protegendo estruturas nervosas e vasculares. Após a divulsão dos tecidos subcutâneos e musculares, incisão com bisturi no periósteo e o descolamento ósseo por meio de ruginas, o ramo mandibular foi exposto, buscando-se a linha de fratura. Com o auxílio do elevador de Ginestet, utilizado com função de abaixador, inserido na incisura mandibular, buscou-se a redução do fragmento ósseo, seguida de osteossíntese com miniplaca compressiva de 2,0 mm (DCP) do sistema AO/ASIF, com quatro perfurações e parafusos bicorticais de titânio, perfurados de maneira cêntrica.

Em algumas situações, com fraturas subcondilares de difícil redução e estabilização dos cotos ósseos para posterior fixação, optou-se pela remoção do fragmento subcondilar, fixação da miniplaca compressiva fora da cavidade articular com perfurações bicorticais, para posterior colocação condilar em sua posição anatômica e adaptação final dos parafusos ${ }^{2,3,4}$ (Figura 2). O bloqueio mandibular no trans operatório foi feito por meio de parafusos ou barra de Erichi.

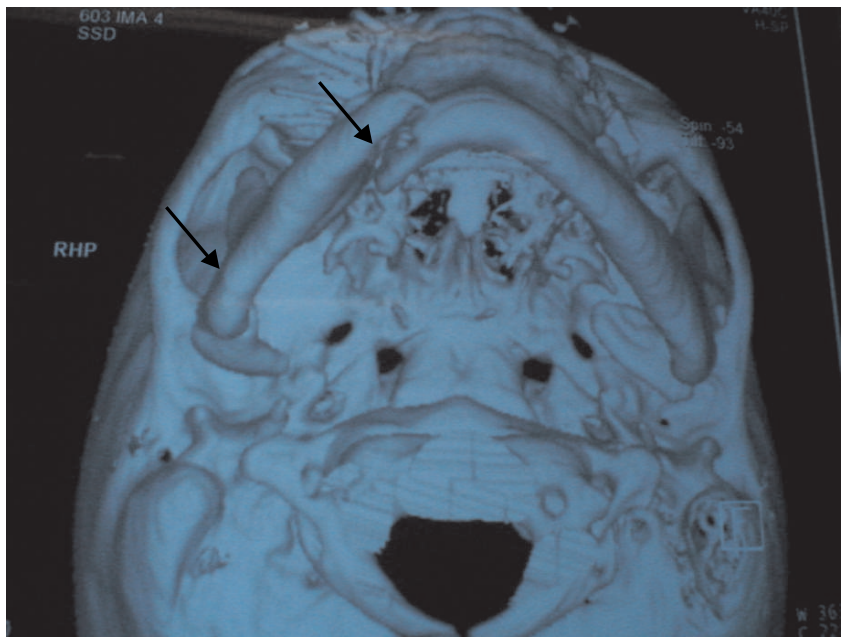

Figura 1 - Tomografia computadorizada tridimensional da face, mostrando fraturas de região subcondilar bilateral, com desvio medial, $e$ de região parassínfisária direita (fonte: casuística da autora).

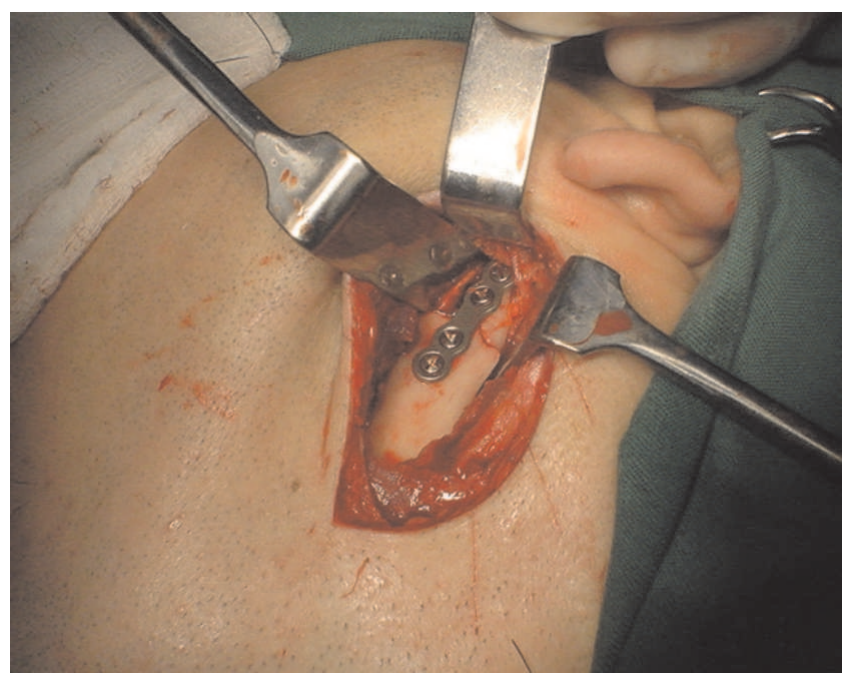

Figura 2 - Colocação do côndilo em posição (redução da fratura)e fixação dos demais parafusos bicorticalmente. (Casuística da autora).

Os pacientes não receberam bloqueio intermaxilar com fios de aço no pós-operatório. Em situações especiais houve a necessidade de bloqueio com tração elástica (elásticos ortodônticos de espessura média, e esféricos) e fisioterapia. Todos os pacientes receberam dieta pastosa no período até 30 a 45 dias do pós operatório, foram avaliados clinicamente e por exames de imagens no $35^{\circ}$ dia de pós-operatório e mensalmente até 12 meses.

Foram avaliados como sinais clínicos e complementares no período pós-operatório: (1)neuropraxia (paralisia facial e parestesias);(2) lateralidade à abertura da boca; (3) amplitude de abertura da boca - mensurada em milímetros com o auxílio de régua ou paquímetro, medida entre incisal de dentes anteriores superiores e incisal de dentes anteriores inferiores. Pacientes apresentando mensurações de 40 a 50mm, foram considerados portadores de ampla abertura bucal. A mensuração foi realizada a partir do $35^{\circ}$ dia de pós-operatório porque resultados inferiores podem ser observados em um período mais próximo do ato cirúrgico sem significar perma- 
nência de alteração; (4) oclusão dentária - classificada em satisfatória de acordo com o retorno ao padrão anterior à cirurgia e comprometida caso houvesse alteração desse padrão e de conformidade com as normas consideradas normais de oclusão; (5) dor - presença de sintomatologia dolorosa com ou sem função mastigatória, classificada em leve, moderada e severa por critérios subjetivos; (6) redução da fratura e estabilidade da osteossíntese - avaliada por exames de imagem com projeções pantomográficas e de Towne modificada.

O sucesso do tratamento cirúrgico foi classificado como parcial quando ocorreu alguma das seguintes situações: presença de neuropraxia, necessidade de tratamento fisioterápico e bloqueio, permanência de desvios ou lateralidade de abertura bucal, amplitude reduzida de abertura bucal (menos de $40 \mathrm{~mm}$ ), presença de dor moderada a intensa ou ausência de redução da fratura e instabilidade da osteossíntese. Todas as demais situações foram classificadas como sucesso total.

Os participantes foram distribuídos em: (a) Grupo 1 fraturas subcondilares bilaterais desviadas para medial, associadas á fraturas de sínfise ou parassínfise mandibular; (b) Grupo 2 - fraturas subcondilares unilaterais com desvios mediais associados a fraturas de corpo ou ângulo mandibular, (c) Grupo 3 - fraturas subcondilares bilaterais sem associação a outras fraturas e deslocamento unilateral e (d) Grupo 4 pacientes portadores de fraturas subcondilares unilaterais com desvios mediais e condilares altas do lado oposto.

A distribuição das variáveis do estudo, em relação aos grupos de estudo está apresentada em tabela de associação com função meramente descritiva. Os resultados referentes à influência das variáveis na resposta terapêutica estão distribuídos em tabelas de associação cruzada. Em relação às variáveis de categoria nominal foi utilizado o teste nãoparamétrico do qui-quadrado para a análise estatística e verificação das hipóteses. Em relação à variável contínua (idade) foi aplicado o teste de diferença de médias (teste t de Student). Em todas as situações foi arbitrado um valor de significância de $5 \%(\mathrm{p}<0,05)$ para refutar a hipótese de nulidade.

\section{RESULTADOS}

Quanto aos resultados, a tabela 1 apresenta a consolidação dos resultados obtidos nas diferentes variáveis em cada um dos grupos de estudo.

Dos sete pacientes com fraturas subcondilares bilaterais com desvios para medial (Tabela 2), associadas à fratura de sínfise ou parassíntese mandibular (Grupo 1), quatro pacientes apresentaram no pós-operatório de 35 dias, abertura bucal ampla, oclusão satisfatória, pouca sintomatologia dolorosa no pós-operatório imediato, e não necessitaram de fisioterapia e de bloqueios intermaxilares com elásticos (Tabelas $1,2,3)$. Dois pacientes necessitaram de bloqueios com elástico e fisioterapia pós-operatória durante 30 dias, por apresentarem desvios mandibulares laterais em movimento de abertura bucal. Um paciente apresentou infecção, edema e algia com 10 dias de pós-operatório, por perda de redução óssea, necessitando de nova intervenção cirúrgica.

Dos cinco pacientes com fraturas subcondilares unilaterais com desvios para medial associados a fraturas de cor- po ou ângulo mandibulares (Grupo 2), quatro obtiveram pósoperatório favorável, ampla abertura bucal, boa oclusão e ausência de parestesia facial (Tabelas 1,2,3,). Uma paciente apresentou quelóide cicatricial, persistindo após 12 meses de pósoperatório e um paciente necessitou de nova intervenção cirúrgica por falha na redução devida a perda de substância óssea em ramo mandibular, provocado por projétil de arma de fogo. Necessitou de enxertia óssea autógena do processo coronóide para obter-se aumento vertical de ramo mandibular. Esse paciente evoluiu no pós-operatório com paralisia facial e foi encaminhado para fisioterapia e drenagem linfática, sem bloqueio intermaxilar, por 60 dias. Com 12 meses de pós-operatório, apresentou diminuição do edema e regeneração parcial do nervo facial. Este foi o único caso de neuropraxia dos 31 pacientes avaliados, correspondendo a $3 \%$ de ocorrência.

Dos nove pacientes com fraturas subcondilares bilaterais com deslocamentos unilaterais mediais e laterais (Grupo 3), dois pacientes portadores de desvios mediais (Tabelas 1,2,3), necessitaram de remoção do fragmento condilar para fixação da placa com parafusos e posterior colocação fragmentar e nova fixação dos parafusos. As fraturas subcondilares do lado oposto foram incompletas, não recebendo fixação interna rígida, nem bloqueios intermaxilares. Sete pacientes, quatro com desvios laterais e três com desvios mediais, necessitaram de bloqueios intermaxilares com elásticos por 35 dias por apresentarem fraturas subcondilares sem desvios, portanto tratadas conservadoramente do lado oposto.

Dos dez pacientes do Grupo 4, seis apresentaram resultados satisfatórios no que diz respeito à abertura bucal, simetria facial e oclusão. Dois pacientes necessitaram de fisioterapias e bloqueios com elásticos por sete dias. Dois pacientes necessitaram de remoção do processo condilar no lado da fratura condilar alta por não ser possível redução e fixação com parafuso, devido a cominução do fragmento condilar. Esses pacientes receberam bloqueios intermaxilares por 45 dias, fisioterapias e colocação de elásticos periódicos. Após 90 dias retornaram a função mastigatória normal, com abertura bucal satisfatória, sem desvios mandibulares laterais.

De acordo o método de classificação de sucesso terapêutico, encontrou-se uma resposta de sucesso total em 19 casos (61\%) e sucesso parcial em 12 casos (39\%).

As tabelas 2 a 3 descrevem a distribuição cruzada entre o sucesso cirúrgico e as variáveis do estudo. Foram observadas diferenças significativas apenas nas variáveis lateralidade (fraturas bilaterais apresentaram uma freqüência maior de sucessos parciais) e desvio da fratura subcondilar (as fraturas com desvio lateral apresentaram uma maior freqüência de sucesso parcial do que as fraturas com desvio medial).

Não foi observada diferença nas médias de idade dos pacientes em relação ao sucesso cirúrgico total ou parcial (33 \pm 15 anos versus $29 \pm 11$ anos, $p=0,453$ ).

\section{DISCUSSÃO}

Pacientes portadores de fraturas subcondilares com desvios significativos, desarmonias faciais e oclusais foram citados como indicações para tratamento cirúrgico. Todavia, 
Tabela 1 - Distribuição das variáveis de estudo nos grupos de estudo.

\begin{tabular}{|c|c|c|c|c|c|}
\hline \multirow[t]{2}{*}{ Variáveis } & \multicolumn{4}{|c|}{ Grupos de estudo } & \multirow[t]{2}{*}{ Total } \\
\hline & 1 & 2 & 3 & 4 & \\
\hline \multicolumn{6}{|l|}{ Gênero } \\
\hline masculino & $\begin{array}{c}2 \\
(28,6 \%)\end{array}$ & $\begin{array}{c}2 \\
(40,0 \%)\end{array}$ & $\begin{array}{c}2 \\
(22,2 \%)\end{array}$ & $\begin{array}{c}9 \\
(90,0 \%)\end{array}$ & $\begin{array}{l}15 \\
(48,4 \%)\end{array}$ \\
\hline feminino & 5 & 3 & 7 & 1 & 16 \\
\hline & $(71,4 \%)$ & $(60,0 \%)$ & $(77,8 \%)$ & $(10,0 \%)$ & $(51,6 \%)$ \\
\hline \multicolumn{6}{|l|}{ Idade } \\
\hline Média \pm DP & $28 \pm 16$ & $36 \pm 14$ & $30 \pm 7$ & $32 \pm 13$ & $31 \pm 13$ \\
\hline Mediana & 25 & 34 & 31 & 30 & 31 \\
\hline Limite inferior & 12 & 22 & 19 & 13 & 12 \\
\hline Limite superior & 57 & 59 & 43 & 63 & 63 \\
\hline \multicolumn{6}{|c|}{ Causa da fratura } \\
\hline queda & $\begin{array}{c}5 \\
(71,4 \%)\end{array}$ & $\begin{array}{l}0 \\
(0,0 \%)\end{array}$ & $\begin{array}{c}5 \\
(55,6 \%)\end{array}$ & $\begin{array}{c}5 \\
(50,0 \%)\end{array}$ & $\begin{array}{l}15 \\
(48,4 \%)\end{array}$ \\
\hline agressão & $\begin{array}{c}2 \\
(28,6 \%)\end{array}$ & $\begin{array}{c}3 \\
(60,0 \%)\end{array}$ & $\begin{array}{c}2 \\
(22,2 \%)\end{array}$ & $\begin{array}{c}3 \\
(30,0 \%)\end{array}$ & $\begin{array}{l}16 \\
(32,3 \%)\end{array}$ \\
\hline acidente & $\begin{array}{l}0 \\
(0,0 \%)\end{array}$ & $\begin{array}{c}2 \\
(40,0 \%)\end{array}$ & $\begin{array}{c}2 \\
(22,2 \%)\end{array}$ & $\begin{array}{c}2 \\
(20,0 \%)\end{array}$ & $\begin{array}{c}6 \\
(19,4 \%)\end{array}$ \\
\hline $\begin{array}{l}\text { Abertura bucal } \\
\text { ampla }\end{array}$ & $\begin{array}{c}4 \\
(57,1 \%)\end{array}$ & $\begin{array}{c}4 \\
(80,0 \%)\end{array}$ & $\begin{array}{c}9 \\
(100,0 \%)\end{array}$ & $\begin{array}{c}10 \\
(100,0 \%)\end{array}$ & $\begin{array}{l}27 \\
(87,1 \%)\end{array}$ \\
\hline limitada & $\begin{array}{c}3 \\
(42,9 \%)\end{array}$ & $\begin{array}{c}1 \\
(20,0 \%)\end{array}$ & $\begin{array}{l}0 \\
(0,0 \%)\end{array}$ & $\begin{array}{l}0 \\
(0,0 \%)\end{array}$ & $\begin{array}{c}4 \\
(12,9 \%)\end{array}$ \\
\hline \multicolumn{6}{|l|}{ Oclusão } \\
\hline satisfatória & $\begin{array}{c}6 \\
(85,7 \%)\end{array}$ & $\begin{array}{c}4 \\
(80,0 \%)\end{array}$ & $\begin{array}{c}9 \\
(100,0 \%)\end{array}$ & $\begin{array}{c}10 \\
(100,0 \%)\end{array}$ & $\begin{array}{l}29 \\
(93,5 \%)\end{array}$ \\
\hline comprometida & $\begin{array}{c}1 \\
(14,3 \%)\end{array}$ & $\begin{array}{c}1 \\
(20,0 \%)\end{array}$ & $\begin{array}{l}0 \\
(0,0 \%)\end{array}$ & $\begin{array}{l}0 \\
(0,0 \%)\end{array}$ & $\begin{array}{l}2 \\
(6,5 \%)\end{array}$ \\
\hline \multicolumn{6}{|l|}{ Dor } \\
\hline leve & $\begin{array}{c}6 \\
(85,7 \%)\end{array}$ & $\begin{array}{c}5 \\
(100,0 \%)\end{array}$ & $\begin{array}{c}9 \\
(100,0 \%)\end{array}$ & $\begin{array}{c}10 \\
(100,0 \%)\end{array}$ & $\begin{array}{l}30 \\
(93,5 \%)\end{array}$ \\
\hline severa & $\begin{array}{c}1 \\
(14,3 \%)\end{array}$ & $\begin{array}{l}0 \\
(0,0 \%)\end{array}$ & $\begin{array}{l}0 \\
(0,0 \%)\end{array}$ & $\begin{array}{l}0 \\
(0,0 \%)\end{array}$ & $\begin{array}{l}1 \\
(6,5 \%)\end{array}$ \\
\hline \multicolumn{6}{|c|}{ Bloqueio pós-operatório } \\
\hline $\operatorname{sim}$ & $\begin{array}{c}2 \\
(28,6 \%)\end{array}$ & $\begin{array}{c}1 \\
(20,0 \%)\end{array}$ & $\begin{array}{c}6 \\
(66,7 \%)\end{array}$ & $\begin{array}{c}2 \\
(20,0 \%)\end{array}$ & $\begin{array}{l}11 \\
(35,5 \%)\end{array}$ \\
\hline não & $\begin{array}{c}5 \\
(71,4 \%)\end{array}$ & $\begin{array}{c}4 \\
(80,0 \%)\end{array}$ & $\begin{array}{c}3 \\
(33,3 \%)\end{array}$ & $\begin{array}{c}8 \\
(20,0 \%)\end{array}$ & $\begin{array}{l}20 \\
(64,5 \%)\end{array}$ \\
\hline
\end{tabular}

há discordância dessa indicação podendo ser feito o tratamento conservador com fisioterapia e bloqueios intermaxilares, cujos resultados são satisfatórios quanto à simetria facial, amplitude de abertura bucal e oclusão dentária ${ }^{13}$. Atribuem a estes resultados, a remodelação condilar relacionada à faixa etária do paciente e a fisioterapia. Todavia, o tratamento conservador apresenta no pós-operatório desvios mandibulares, mordidas abertas anteriores e desordens oclusais ${ }^{7}$. Em estudos comparativos entre tratamentos fechados e abertos para pacientes portadores de fraturas condilares com desvios diversos autores ${ }^{14-19}$, relatam resultados pós-operatórios satisfatórios, nos pacientes tratados cirurgicamente, referindo-se à simetria facial, amplitude de abertura bucal, oclusão dentária semelhante à oclusão anterior ao trauma, movimentação mandibular de abertura, lateralidade e protrusão, devolvendo ao paciente a normalização de suas atividades funcionais.

A redução das fraturas subcondilares pode ser feita por endoscopia com abordagem intra-oral ${ }^{8}$. A abordagem retromandibular de Hinds, descrita neste estudo é também citada em vários outros ${ }^{15,20-23}$, porque apresenta facilidade de acesso à fratura e pouca morbidade, comparada a outras abor- 
Tabela 2 - Distribuição das variáveis do estudo de acordo com o sucesso cirúrgico.

\begin{tabular}{|c|c|c|c|}
\hline \multirow[t]{2}{*}{ Variável } & \multicolumn{2}{|c|}{ Sucesso cirúrgico } & \multirow[t]{2}{*}{ Total } \\
\hline & Total n (\%) & Parcial n (\%) & \\
\hline \multicolumn{4}{|l|}{ Gênero ${ }^{\mathrm{NS}}$} \\
\hline masculino & $10(52,6 \%)$ & $5(41,7 \%)$ & $15(48,4 \%)$ \\
\hline feminino & $9(47,4 \%)$ & $7(58,3 \%)$ & $16(51,6 \%)$ \\
\hline \multicolumn{4}{|l|}{ Grupo $^{\mathrm{NS}}$} \\
\hline Grupo 1 & $4(21,1 \%)$ & $3(25,0 \%)$ & $7(22,6 \%)$ \\
\hline Grupo 2 & $4(21,1 \%)$ & $1 \quad(8,3 \%)$ & $5(16,1 \%)$ \\
\hline Grupo 3 & $3(15,8 \%)$ & $6(50,0 \%)$ & $9(29,0 \%)$ \\
\hline Grupo 4 & $8(42,1 \%)$ & $2(16,7 \%)$ & $10(32,3 \%)$ \\
\hline \multicolumn{4}{|l|}{ Causa da lesão ${ }^{\mathrm{NS}}$} \\
\hline queda & $10(52,6 \%)$ & $5(41,7 \%)$ & $17(48,4 \%)$ \\
\hline agressão & $5(26,3 \%)$ & $5(41,7 \%)$ & $10(32,3 \%)$ \\
\hline acidente & $4(21,1 \%)$ & $2(16,7 \%)$ & $6(19,4 \%)$ \\
\hline \multicolumn{4}{|c|}{ Lateralidade da lesão ${ }^{\mathrm{NS}}$} \\
\hline unilateral & $12(63,2 \%)$ & $3(25,0 \%)$ & $15(48,4 \%)$ \\
\hline bilateral & $7(36,8 \%)$ & $9(75,0 \%)$ & $16(51,6 \%)$ \\
\hline \multicolumn{4}{|l|}{ Fratura associada $^{\mathrm{NS}}$} \\
\hline única & $8(42,1 \%)$ & $4(33,3 \%)$ & $12(38,7 \%)$ \\
\hline múltipla & $8(42,1 \%)$ & $2(16,7 \%)$ & $10(32,3 \%)$ \\
\hline ausente & $3(15,8 \%)$ & $6(50,0 \%)$ & $9(29,0 \%)$ \\
\hline \multicolumn{4}{|c|}{ Localização da fratura associada ${ }^{\mathrm{NS}}$} \\
\hline ângulo mandibular & $2(10,5 \%)$ & $1 \quad(8,3 \%)$ & $3(9,7 \%)$ \\
\hline corpo mandibular & $2(10,5 \%)$ & $0 \quad(0,0 \%)$ & $2(6,5 \%)$ \\
\hline sínfise + mandíbula & $8(42,1 \%)$ & $2(16,7 \%)$ & $10(32,3 \%)$ \\
\hline ausente & $3(15,8 \%)$ & $6(50,0 \%)$ & $9(29,0 \%)$ \\
\hline
\end{tabular}

NS = diferença não significativa $(p>0,005)$.

dagens. Nossos resultados diferem da literatura ${ }^{20}$, no que diz respeito a bloqueios intermaxilares pós-operatórios utilizando-se a abordagem retromandibular de Hinds, pois nenhum de nossos pacientes necessitou de bloqueios intermaxilares com elásticos acompanhados por 22 meses de pós-operatório. Os resultados dos autores diferem do presente estudo demonstrando que $39 \%$ dos pacientes necessitaram de bloqueios com elásticos no pós-operatório, sendo classificados como sucesso parcial. Estes resultados foram atribuídos as variáveis dos grupos apresentados. Houve discordâncias dos resultados ${ }^{23,24}$, no que diz respeito a abordagens, descrevem a abordagem pré-auricular como a mais indicada para a redução das fraturas condilares por apresentar uma proximidade maior da região condilar e, portanto, ao traço de fratura. Essa incisão, segundo esses autores, proporciona melhor condição de realização da redução e osteossíntese; porém, apresenta gran-

Tabela 3 - Distribuição da variável desvio da lesão de acordo com o sucesso cirúrgico.

\begin{tabular}{|c|c|c|c|}
\hline \multirow[t]{2}{*}{ Desvio da lesão * } & \multicolumn{2}{|c|}{ Sucesso cirúrgico } & \multirow[t]{2}{*}{ Total } \\
\hline & Total & Parcial & \\
\hline medial & $\begin{array}{l}11 \\
(57,9 \%)\end{array}$ & $\begin{array}{c}6 \\
(50,0 \%)\end{array}$ & $\begin{array}{l}17 \\
(54,8 \%)\end{array}$ \\
\hline lateral & $\begin{array}{l}0 \\
(0,0 \%)\end{array}$ & $\begin{array}{c}4 \\
(33,3 \%)\end{array}$ & $\begin{array}{c}4 \\
(12,9 \%)\end{array}$ \\
\hline ausente & $\begin{array}{c}8 \\
(42,1 \%)\end{array}$ & $\begin{array}{c}2 \\
(16,7 \%)\end{array}$ & $\begin{array}{l}10 \\
(32,3 \%)\end{array}$ \\
\hline Total & $\begin{array}{c}19 \\
(100,0 \%)\end{array}$ & $\begin{array}{c}12 \\
(100,0 \%)\end{array}$ & $\begin{array}{c}31 \\
(100,0 \%)\end{array}$ \\
\hline
\end{tabular}

* = diferenças significativas

$x^{2}=7,892$ 
de morbidade, sendo melhor indicada nas fraturas condilares altas ou intra- capsulares ${ }^{4}$.

Este estudo, apesar de não ser sobre indicações de tratamento para fraturas subcondilares, assemelha-se ao de Aguiar ${ }^{4}$, em 1984, quando indicaram tratamento cirúrgico para fraturas condilares com deslocamentos em: adultos edêntulos portadores de fraturas bilaterais com grandes desvios e conseqüentemente encurtamento de ramos e, por conseguinte mordida aberta anterior e em crianças, se o grau de separação dos cotos ósseos for de tal magnitude que possa afetar o centro de crescimento ósseo.

Relacionando-se aos métodos de fixação interna rígida, há relato semelhante ${ }^{25}$, ao resultado do nosso estudo, afirmando que a melhor estabilidade é conseguida com placas de titânio e parafusos bicorticais, perpendiculares ao traço de fratura, sistema AO/ASIF da Synthes, podendo ser realizada com uma placa compressiva $2.0 \mathrm{~mm}$. Outros autores ${ }^{16,26}$, todavia, estabeleceram que a melhor estabilidade é conseguida com aplicação de duas miniplacas $2.0 \mathrm{~mm}$ e parafusos bicorticais.

Este estudo retrospectivo não comparou a estabilidade dos materiais de fixação, somente demonstrando que a fixação empregada apresentou resistência e estabilidade num pós-operatório de 12 meses, devolvendo ao paciente a função e a harmonia mandibular. Com relação a amplitude de abertura bucal, simetria facial, harmonia oclusal e neuropraxias há trabalhos cujo resultado é semelhante ao nosso ${ }^{27}$.

Assim como neste estudo, Aguiar ${ }^{4}$, adotaram a abordagem paralela à borda posterior do ramo mandibular, preconizada por Hinds ${ }^{28}$ para cirurgia ortognática, pois a consideraram uma abordagem intermediária entre a pré-auricular e a submandibular, justificando que proporciona uma ampla visão do campo operatório, além de apresentar menor morbidade com relação ao nervo facial e dos vasos sanguíneos da região. Outro trabalho ${ }^{29}$, demonstrou resultado semelhante ao nosso, quando afirmam que a abordagem retromandibular apresenta menores complicações operatórias e, portanto, menor morbidade. Apesar deste não ser um estudo comparativo de abordagens, apresentamos um resultado de $61 \%$ de sucesso total nos pacientes intervidos com o acesso retromandibular de Hinds.

De acordo com os achados do estudo pode-se concluir que, na amostra estudada, a abordagem retromandibular de Hinds para tratamento das fraturas subcondilares, na maioria dos pacientes teve sucesso total, proporcionando o retorno às atividades diárias, uma perfeita harmonia estética (sem desvios mandibulares), e ampla abertura bucal.

\begin{abstract}
Background: The absence of definitive studies about more suitable surgical approach for sub-condylar fractures supports an analysis on Hinds retromandibular approach along the treatment response assessment. Method: It was an observational, transversal, retrospective protocol including 31 cases divided in four groups accordingly to the laterality, fracture dislocation, and the association of fractures in other sites. The therapeutic success has been classified according to the need of physiotherapeutic treatment and blockage; dislocation persistence or laterality of buccal opening; reduced amplitude of buccal opening (less than $40 \mathrm{~mm}$ ); presence of moderate to severe pain; or absence of fracture reduction and instability of osteosynthesis. To assess the determinant success factors, the variables gender, age, fracture cause, laterality, dislocation, associated fractures, and fractures sites have been included. The statistic method consisted of cross-association tables with analysis using the non-parametric test, chi-square. In each situation, a significant value of $5 \%(p<0.05)$ was attributed. Results: We obtained total response success in 19 cases (61\%) and partial success in 12 cases (39\%). We found no association between surgical response variables such as gender, age, fracture cause, and presence of an associated fracture. Lateral dislocations and injury bilateralism have shown a higher frequency of partial success cases. Conclusion: The Hinds retromandibular approach showed therapeutic success in most cases with a comprehensive access for reduction of sub-condylar fractures, stable fixation and with no postoperative complications.
\end{abstract}

Key words: Condylar fracture of mandible; Surgery.

\section{REFERÊNCIAS}

1. Filho LI, Pavan AJ, Camarini ET, Tondin GM. Tratamento das fraturas de côndilo mandibular: cirúrgico ou conservador? Rev Cir Traumatol Buco-Maxilo-Fac. 2005; 4:17-22.

2. Valiat R, Araújo AC, Spironello JA, Silva FM, Silva DS, Lemes RS. Comparativo no tratamento de fraturas baixas de côndilo com uma ou duas osteossínteses a fio de aço. Rev Bras Cir Implant.1998;5(4):23-7.

3. Lindahl L. Condylar fractures of the mandible. I. Classification and relation to age, occlusion, and concomitant injuries of teeth and teeth-supporting structures, and fractures of the mandibular body. Int J Oral Surg. 1977 Feb;6(1):12-21.

4. Aguiar SA. Traumatologia buco-maxilo-facial. In: Canto Pereira L C. Odontologia Hospitalar. Ed Santos; 1984:155-75.
5. Marker P, Nielsen A, Bastian HL. Fractures of the mandibular condyle. Part 2: results of treatment of 348 patients.Fractures of the mandibular condyle. Part 2: results of 348 patients. Br J Oral Maxillofac Surg. 2000 Oct;38(5):422-6.

6. Hammer B, Schier P, Prein J. Osteosynthesis of condylar neck fractures: a review of 30 patients. Br J Oral Maxillofac Surg. 1997 Aug;35(4):288-91. .

7. Thorén H, Hallikainen D, Iizuka T, Lindqvist C. Condylar process fractures in children: a follow-up study of fractures with total dislocation of the condyle from the glenoid fossa. J Oral Maxillofac Surg. 2001 Jul;59(7):768-73; discussion 773-4.

8. Lee C, Stiebel M, Young DM. Cranial nerve VII region of the traumatized facial skeleton: optimizing fracture repair with the endoscope. J Trauma. 2000 Mar;48(3):423-31; discussion 431-2. 
9. Sugiura T, Yamamoto K, Murakami K, Sugimura M. A comparative evaluation of osteosynthesis with lag screws, miniplates, or Kirschner wires for mandibular condylar process fractures. J Oral Maxillofac Surg. 2001 Oct;59(10):1161-8; discussion 1169-70.

10. Ellis E 3rd, Throckmorton GS, Palmieri C. Open treatment of condylar process fractures: assessment of adequacy of repositioning and maintenance of stability. J Oral Maxillofac Surg. 2000 Jan;58(1):27-34; discussion 35.

11. Lauer G, Schmelzeisen R. Endoscope-assisted fixation of mandibular condylar process fractures. J Oral Maxillofac Surg. 1999 Jan;57(1):36-9; discussion 39-40.

12. Ellis E 3rd, McFadden D, Simon P, Throckmorton G. Surgical complications with open treatment of mandibular condylar process fractures. J Oral Maxillofac Surg. 2000 Sep;58(9):950-8. Comment in: J Oral Maxillofac Surg. 2001 Jun;59(6):715.

13. Nørholt SE, Krishnan V, Sindet-Pedersen S, Jensen I. Pediatric condylar fractures: a long-term follow-up study of 55 patients. $J$ Oral Maxillofac Surg. 1993 Dec;51(12):1302-10.

14. Zhang X, Obeid G. A comparative study of the treatment of unilateral fractured and dislocated mandibular condyles in the rabbit. J Oral Maxillofac Surg. 1991 Nov;49(11):1181-90.

15. Ellis E 3rd, Throckmorton GS, Palmieri C. Open treatment of condylar process fractures: assessment of adequacy of repositioning and maintenance of stability. J Oral Maxillofac Surg. 2000 Jan;58(1):27-34; discussion 35.

16. Throckmorton GS, Ellis E 3rd, Hayasaki H. Masticatory motion after surgical or nonsurgical treatment for unilateral fractures of the mandibular condylar process. J Oral Maxillofac Surg. 2004 Feb;62(2):127-38.

17. Palmieri C, Ellis E 3rd, Throckmorton G. Mandibular motion after closed and open treatment of unilateral mandibular condylar process fractures. J Oral Maxillofac Surg. 1999 Jul;57(7):76475; discussion 775-6.

18. Yang WG, Chen CT, Tsay PK, Chen YR. Functional results of unilateral mandibular condylar process fractures after open and closed treatment. J Trauma. 2002 Mar;52(3):498-503

19. Haug RH, Assael LA. Outcomes of open versus closed treatment of mandibular subcondylar fractures. J Oral Maxillofac Surg. 2001 Apr;59(4):370-5; discussion 375-6.

20. Choi BH, Yoo JH. Open reduction of condylar neck fractures with exposure of the facial nerve. Oral Surg Oral Med Oral Pathol Oral Radiol Endod. 1999 Sep;88(3):292-6.
21. Hyde N, Manisali M, Aghabeigi B, Sneddon K, Newman L. The role of open reduction and internal fixation in unilateral fractures of the mandibular condyle: a prospective study. Br J Oral Maxillofac Surg. 2002 Feb;40(1):19-22.

22. Landes CA, Lipphardt R. Prospective evaluation of a pragmatic treatment rationale: open reduction and internal fixation of displaced and dislocated condyle and condylar head fractures and closed reduction of non-displaced, non-dislocated fractures Part II: high condylar and condylar head fractures. Int J Oral Maxillofac Surg. 2006 Feb;35(2):115-26.

23. Takenoshita Y, Ishibashi H, Oka M. Comparison of functional recovery after nonsurgical and surgical treatment of condylar fractures. J Oral Maxillofac Surg. 1990 Nov;48(11):1191-5.

24. Worsaae N, Thorn JJ. Surgical versus nonsurgical treatment of unilateral dislocated low subcondylar fractures: a clinical study of 52 cases.. J Oral Maxillofac Surg. 1994 Apr;52(4):353-60; discussion 360-1

25. Hammer B, Schier P, Prein J. Osteosynthesis of condylar neck fractures: a review of 30 patients. Br J Oral Maxillofac Surg. 1997 Aug;35(4):288-91.

26. Choi BH, Yi CK, Yoo JH. Clinical evaluation of 3 types of plate osteosynthesis for fixation of condylar neck fractures. J Oral Maxillofac Surg. 2001 Jul;59(7):734-7; discussion 738.

27. Ellis E 3rd, Simon P, Throckmorton GS. Occlusal results after open or closed treatment of fractures of the mandibular condylar process. J Oral Maxillofac Surg. 2000 Mar;58(3):260-8

28. Hinds EC, Kent JN. Tratamiento quirúrgico de las anomalias de desarrollos de los maxilares. Ed Labor: Barcelona; 1974.p.82.

29. Ellis E 3rd, McFadden D, Simon P, Throckmorton G. Surgical complications with open treatment of mandibular condylar process fractures. J Oral Maxillofac Surg. 2000 Sep;58(9):950-8

Como citar este artigo:

Goto AAA, Aguiar SA, Denardin OVP, Rapoport A. Tratamento cirúrgico das fraturas subcondilares: avaliação da abordagem retromandibular de hinds. Rev Col Bras Cir. [periódico na Internet] 2007 Set-Out; 34(5). Disponível em URL: www.scielo.br/rcbc

Endereço para correspondência:

Prof. Dr. Abrão Rapoport

Rua Iramaia, nº 136 - Jd. Europa - São Paulo

01450-020 - São Paulo - SP

E-mail: arapoport@ terra.com.br 